\title{
A non-cyclic, locally free, free-by-cyclic group all of whose finite factor groups are cyclic
}

\section{Gilbert Baumslag}

We construct a group $G$ with the properties stated in the title.

We construct here a group $G$ with the properties described in the title of this note. The properties of $G$ should be viewed in the context of the following theorem:

A finitely generated cyclic extension of a free group is residually finite [1].

We construct $G$ as a direct limit of free groups $G_{i}=\left\langle a_{i}, b_{i}\right\rangle$ of rank two. To this end let $\phi_{i}: G_{i} \rightarrow G_{i+1}$ be defined as follows:

$$
a_{i} \phi_{i}=b_{i+1}^{-(i+1) !} a_{i+1}^{-1} b_{i+1}^{(i+1) !} a_{i+1}, b_{i} \phi_{i}=b_{i+1}, i=1,2, \ldots
$$

It follows easily that $\phi_{i}$ is a monomorphism. The groups $G_{i}$ together with the monomorphisms $\phi_{i}$ constitute a direct system. The direct limit of this system is the desired group $G$. As usual we identify, for example, $a_{i}$ with its image $a_{i} \phi_{i}$. Then $G$ is the union of its subgroups $G_{i}$. Moreover, if we put $b_{1}=b$, then $b_{i}=b$ for all $i$.

In order to see that $G$ has the desired properties, let $N_{i}$ be the normal closure in $G_{i}$ of $a_{i}$. Then it is not hard to show that $N_{i}$ is a

Received 16 November 1971. The author gratefully acknowledges support from the National Science Foundation. 
free factor of $N_{i+1}$. Therefore the union $N$ of these subgroups $N_{i}$ is a free group. But $N$ is the normal subgroup of $G$ generated by the elements $a_{1}, a_{2}, \ldots$. It follows easily that $G / N$ is infinite cyclic on $b N$, which means that $G$ is free-by-cyclic as claimed. In addition $G$ is locally free because it is an ascending union of free groups. It remains only to show that every finite factor group of $G$ is cyclic.

Let $K$ be a normal subgroup of $G$ of finite index, $n$ say. Then, working modulo $K$, we have

$$
a_{n} \equiv b_{n+1}^{-(n+1) !} a_{n+1}^{-1} b_{n+1}^{(n+1) !} a_{n+1} \equiv a_{n+1}^{-1} a_{n+1}=1 \quad(K) \text {. }
$$

This implies that $N_{n} \leq K$. Since $N_{i} \leq N_{n}$ when $i \leq n$, it follows that $N_{i} \leq K$, for $i \leq n$. However a similar inductive argument shows that $N_{i} \leq K$ for every $i$. So $N \leq K$. Therefore $G / K$ is cyclic, as claimed. This completes the proof that $G$ is a group of the desired type.

\section{Reference}

[1] Gilbert Baumslag, "A non-cyclic one-relator group all of whose finite quotients are cyclic", J. Austral. Math. Soc. 10 (1969), 497-498.

Rice University,

Houston,

Texas,

USA. 